\title{
Comparison of Pancreatic Microcirculation Profiles in Spontaneously Hypertensive Rats and Wistar-Kyoto Rats by Laser Doppler and Wavelet Transform Analysis
}

\author{
Xiaohong SONG ${ }^{1 *}$, Yuan LI $^{1 *}$, Bing WANG ${ }^{1}$, Mingming LIU ${ }^{1,2}$, Jian ZHANG ${ }^{1,2}$, Ailing LI $^{1}$, \\ Honggang ZHANG ${ }^{1}$, Ruijuan XIU ${ }^{1}$ \\ *These authors contributed equally to this work \\ ${ }^{1}$ Institute of Microcirculation, Chinese Academy of Medical Sciences and Peking Union Medical \\ College, Beijing, China, ${ }^{2}$ Diabetes Research Center, Chinese Academy of Medical Sciences and \\ Peking Union Medical College, Beijing, China
}

Received February 5, 2019

Accepted August 25, 2020

Epub Ahead of Print November 2, 2020

\begin{abstract}
Summary
Pancreatic microcirculatory dysfunction emerged as a novel mechanism in the development of hypertension. However, the changes of pancreatic microcirculation profiles in hypertension remain unknown. Pancreatic microcirculatory blood distribution pattern and microvascular vasomotion of spontaneously hypertensive rats (SHRs) and Wistar Kyoto rats (WKYs) were determined by laser Doppler. Wavelet transform analysis was performed to convert micro-hemodynamic signals into timefrequency domains, based on which amplitude spectral scalograms were constructed. The amplitudes of characteristic oscillators were compared between SHRs and WKYs. The expression of eNOS was determined by immunohistochemistry, and plasma nitrite/nitrate levels were measured by Griess reaction. Additionally, endothelin-1, malondialdehyde, superoxide dismutase and interleukin- 6 were determined by enzyme-linked immunosorbent assay. SHRs exhibited a lower scale blood distribution pattern with decreased average blood perfusion, frequency and amplitude. Wavelet transform spectral analysis revealed significantly reduced amplitudes of endothelial oscillators. Besides reduced expression of eNOS, the blood microcirculatory chemistry complements micro-hemodynamic profiles as demonstrated by an increase in plasma nitrite/nitrate, endothelin-1, malondialdehyde, interleukin- 6 and a decrease of superoxide dismutase in SHRs. Here, we described abnormal pancreatic microcirculation profiles in SHRs, including disarranged blood distribution pattern, impaired microvascular vasomotion and reduced amplitudes of endothelial oscillators.
\end{abstract}

\section{Key words}

Pancreatic microcirculation profiles - Distribution pattern • Microvascular vasomotion - Amplitude • Spontaneously hypertensive rats

\section{Corresponding author}

Mingming Liu and Honggang Zhang, Institute of Microcirculation, Chinese Academy of Medical Sciences and Peking Union Medical College, No.5 Dong Dan San Tiao, Dongcheng District, Beijing, 100005, China, E-mail: mingmingliu@imc.pumc.edu.cn, zhanghg1966126@163.com

\section{Introduction}

Hypertension, characterized by elevated blood pressure and irregular peripheral vascular resistance regulation, is one of the most widespread cardiovascular diseases (DALYs and Collaborators 2016). Generally, hypertension is not a single disease and instead encompasses various clinical features, such as hyperglycemia and insulin resistance. The incidence and progression of cardiovascular events and microvascular complications are associated with hypertension. However, the pathogenesis of hypertension has not fully clarified yet.

There is growing attention on the connection between microcirculation and hypertension recently. Pancreatic microcirculatory dysfunction emerged as 
a novel pathogenesis of hypertension. It has been reported that uncontrolled proteolytic receptors cleavage occurred in hypertensive condition, which leading to the cellular and systemic dysfunction (Chan and Schmid-Schonbein 2019, Delano and Schmid-Schonbein 2008, StruijkerBoudier et al. 2007), even end-organic damage (Delano et al. 2010). Subsequently, pancreas has been proved as a major source of those digestive enzymes and degrading protease activities occurred in the microcirculation (Chan and Schmid-Schonbein 2019). These lines of evidence highlight the importance of pancreatic microcirculation in hypertension.

Microcirculation is essential for providing nutrients and removing metabolites in response to metabolic demands and fluctuated hydrostatic pressure. There is an agreement that microcirculation participates in maintaining the physiological function of organs (De Boer et al. 2012). In this respect, microcirculation dysfunction would be involved in the pathogenesis of hypertension (Levy et al. 2001). Furthermore, as a characteristic phenomenon of microcirculation profiles, microvascular vasomotion regulates blood flow perfusion and distribution in organs (Gutterman et al. 2016). Several studies have reported impairments of the vasomotion (Kobayashi et al. 2005) and microvasculature in hypertensive individuals (Debbabi et al. 2006, Gkaliagkousi et al. 2015, Tousoulis et al. 2006, Tran and Schmid-Schonbein 2007), which confirm the link between pathological disturbed microcirculation and hypertensive damage. The possibility that digestive enzymes leak out of the pancreas has given rise to hypotheses as that of the abnormality of pancreatic microcirculation.

Analyses of pancreatic microcirculation profiles are the reasonable strategy to investigate the changes of pancreatic microcirculation. We have established a method to assess pancreatic microcirculation profiles by micro-hemodynamic and microcirculatory blood distribution pattern. As far as we know, there was little information on the pancreatic microcirculation profiles in hypertension. Therefore, the aim of the current study was to investigate the difference of pancreatic microcirculation profiles between spontaneously hypertensive rats (SHRs) and their normotensive control Wistar Kyoto rats (WKYs).

\section{Methods}

Animals

This study was approved by the Institutional Animal Care and Use Committee at the Institute of Microcirculation, Chinese Academy of Medical Sciences (CAMS), in accordance with the guidelines for the Care and Use of Laboratory Animals (IACUC-201709). The SHR is a commonly used model of hypertension. These rats develop increased blood pressure beginning at eight to nine weeks of age with blood pressure at $160-170 \mathrm{mmHg}$ and reach a stable level of hypertension at $185-190 \mathrm{~mm} \mathrm{Hg}$ during 16 to 28 weeks of age. Eight- week-old male SHRs and its normotensive control WKYs ( $n=6$ each group) were provided by the Institute of Laboratory Animal Sciences (CAMS, Beijing, China). Rats were housed separately in the cages of the animal room (temperature $22{ }^{\circ} \mathrm{C}$, humidity $55 \%-70 \%$ ) under a $12 \mathrm{~h}$ light/dark cycle and were fed with standard laboratory diet and water ad libitum. The weight of all rats was recorded and general information (including age, gender, body weight and blood pressure) was listed in Table 1.

Table 1. General information of WKYs and SHRs.

\begin{tabular}{lcc}
\hline & WKYs & SHRs \\
\hline Age (week) & 8 & 8 \\
Gender (male/female) & $6 / 0$ & $6 / 0$ \\
Body weight (g) & $202 \pm 2.8$ & $172.4 \pm 1.38^{*}$ \\
Blood pressure (mmHg) & & \\
HR & $370.3 \pm 1.4$ & $422.3 \pm 4.2$ \\
SBP & $118.2 \pm 2.5$ & $167 \pm 3.2^{*}$ \\
MAP & $94.2 \pm 3.0$ & $134.2 \pm 3.5^{*}$ \\
DBP & $82.3 \pm 2.5$ & $118 \pm 3.9^{*}$ \\
\hline
\end{tabular}

Data were expressed as the mean \pm S.E.M. HR, heart rate; SBP, systolic blood pressure; DBP, diastolic blood pressure; MAP, mean arterial pressure. ${ }^{*} P<0.01$ compared with WKYs. 


\section{Measurement of blood pressure}

A Biopac MP 150 system and AcqKnowledge software (BIOPAC) were employed to measure blood pressure of rats. After acclimatization, rats were anesthetized by $2 \%$ inhaled isoflurane in a $50 \%$ mixture of oxygen and then fixed in a lateral decubitus position. A 10-mm midline longitudinal incision was cut to expose common carotid artery and external carotid artery. The distal segment of common carotid artery was ligated permanently while a temporary ligation was made at proximal segment to provide a blood flow-free segment in common carotid artery. Then, a micro-catheter, combined with pressure transducer and transmitter, was advanced into the carotid artery by retrograde way. The micro-catheter was rotated and secured with tip oriented cephalad. Heart rate (HR) and blood pressure including systolic blood pressure (SBP), diastolic blood pressure (DBP) and mean arterial pressure (MAP) of rats were measured and recorded respectively.

\section{Assessment of pancreatic microcirculation profiles}

The pancreatic microcirculation profiles of SHRs and WKYs were detected according to the Doppler frequency shift principle with a dual-channel laser Doppler blood perfusion monitoring system (Moor Instrument, Ltd., Axminster, UK) as previously described (Liu et al. 2018). Briefly, after 10 min acclimatization, rats were anesthetized by $2 \%$ inhaled isoflurane in a $50 \%$ mixture of oxygen. A designed incision was made around medioventral line to expose pancreas. Laser Doppler signals of microvascular blood perfusion were measured by VP4 probe (Moor Instrument) and analyzed by Moor software (Moor VMS PC 3.1, Moor Instruments). To depict blood distribution pattern, changes of microcirculatory blood perfusion during contraction and dilation were illustrated in a scatter plot. The average blood perfusion was calculated as the microvascular blood perfusion divided by mins, while velocity was captured according to the changes of scattered light intensity of standard micro-particles. Furthermore, the frequency and amplitude of microvascular vasomotion were calculated as the number of peaks occurred per min and the difference of perfusion unit $(\triangle \mathrm{PU})$ between minimum $\mathrm{PU}$ and maximum $\mathrm{PU}$ respectively.

\section{Wavelet transform spectral analysis}

Wavelet transform spectral analysis, revealing the contribution of specific biological oscillators in microcirculation to the nonlinear dynamic changes of microvascular blood flow, was performed to convert microcirculatory perfusion signals into time-frequency domains. Considering the common used frequency intervals applied for investigating microcirculatory function of human and experimental animals (Aleksandrin et al. 2018, Mastantuono et al. 2017, Popa et al. 2015), the entire frequency derived from laser Doppler signals was divided into a set of frequency bands that each of them contains a single peak: $2 \sim 5 \mathrm{~Hz}$, $0.4 \sim 2 \mathrm{~Hz}, 0.15 \sim 0.4 \mathrm{~Hz}, 0.04 \sim 0.15 \mathrm{~Hz}$ and $0.01 \sim 0.04 \mathrm{~Hz}$ (Aleksandrin et al. 2018), attributing to cardiac, respiratory, myogenic, neurogenic and endothelial oscillators respectively. Due to microvascular endothelial cells are the most important component of microcirculation and the functional executing unit of microvascular vasomotion, in the current study, we separated these frequency bands into endothelial (NO-dependent and NO-independent endothelial) oscillators (Lapi et al. 2017, Stefanovska et al. 1999) and non-endothelial (cardiac, respiratory, myogenic, neurogenic) oscillators.

The Morlet wavelet (Lancaster et al. 2015) was scaled to provide a Gaussian window which is shifted along the time and frequency domains (Smirni et al. 2018), and wavelet amplitudes were calculated by averaging the wavelet coefficients to represent timefrequency spectral characteristic of those oscillators. Furthermore, a three-dimensional (3-D) amplitude spectral scalogram was constructed based on the wavelet transformed micro-hemodynamic data. Variants including time (s), frequency $(\mathrm{Hz})$ and spectral amplitude (AU) were located in the coordinates respectively to show coordinated time-frequency resolution. The amplitudes of six oscillators were compared between WKYs and SHRs.

\section{Immunohistochemistry}

Immunostaining was used to evaluate expression of endothelial nitric oxide synthase (eNOS). Briefly, to inhibit endogenous peroxidase, deparaffinized pancreas sections were treated with $3 \%$ hydrogen peroxide, followed by blocking with $3 \%$ bovine serum albumin in PBS (TBD Science Technology, Tianjin, China). Primary antibody against eNOS (1: 50; Santa Cruz Biotechnology) was incubated in blocking buffer overnight at $4{ }^{\circ} \mathrm{C}$. After rinsing, sections were incubated with horseradish peroxidase conjugated secondary antibody (Zhongshan Golden Bridge Biotechnology, Beijing, China). Slides were washed and mounted prior to 
observation. Positive staining of eNOS was captured using Leica DFC450 microscope (Leica Microsystems, Leitz, Germany).

\section{Enzyme-linked immunosorbent assays (ELISAs)}

Plasma nitrite/nitrate concentrations of WKYs and SHRs were measured using a Griess reaction assay kit (R\&D Systems, MN, USA). Plasma superoxide dismutase (SOD) (Blue Gene Biotech, Shanghai, China), malondialdehyde (MDA) (Blue Gene Biotech), interleukin-6 (IL-6) (Blue Gene Biotech) and endothelin1 (ET-1) (R\&D Systems) levels were determined by the ELISAs kits following the manufacturer's protocols. The optical density was recorded by microplate reader
(Thermo Scientific ${ }^{\mathrm{TM}}$ Multiskan ${ }^{\mathrm{TM}}$ GO, MA, USA).

\section{Statistical analysis}

All microvascular blood perfusion signals and wavelet transform spectral data were presented as means \pm standard errors of the means (S.E.M.) and SPSS version 21.0 (SPSS Inc., Chicago, IL, USA) was used to perform the statistical analysis. Comparisons of pancreatic microcirculation profiles were conducted by Student $t$-test and $P$ value of less than 0.05 was considered as statistically significant. The correlations were established by calculating the Pearson's correlation coefficient $(r)$, and were considered relevant for associated $P<0.05$ and values of $r>0.3$ or $<-0.3$.
A
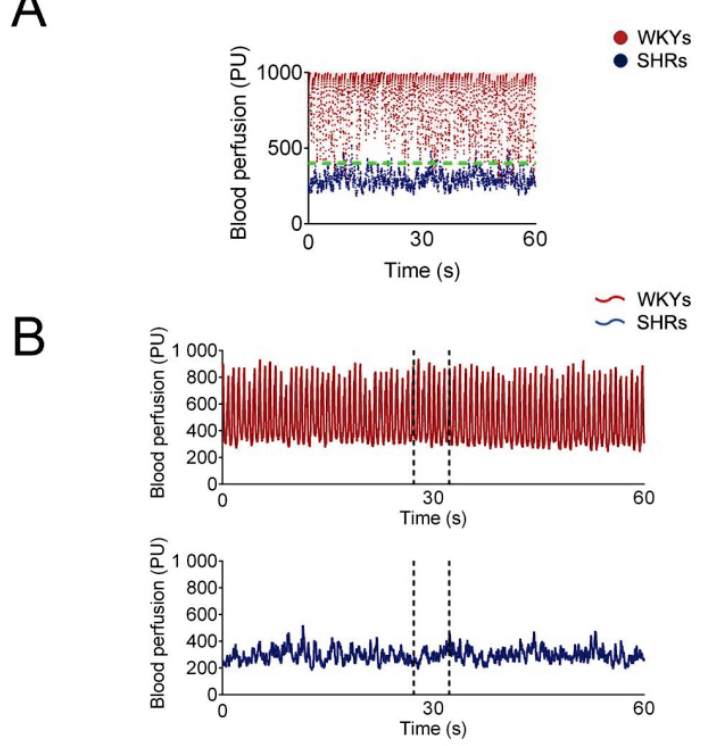

E

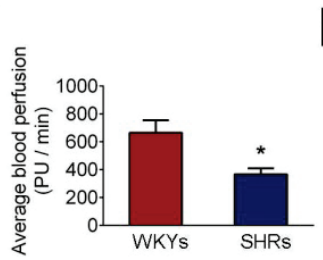

F

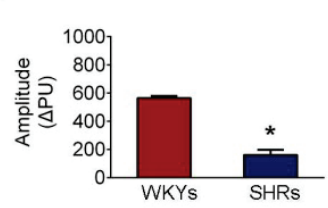

C
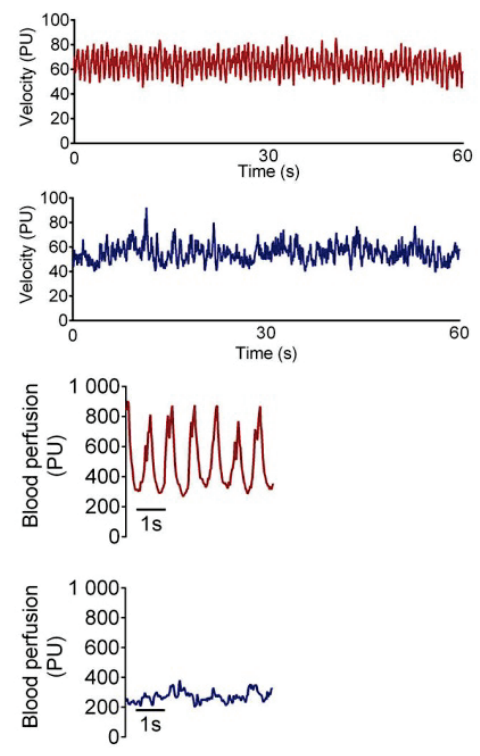

G

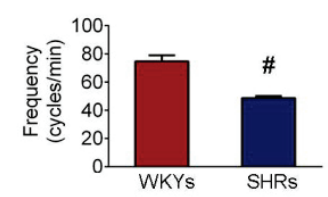

$\mathrm{H}$

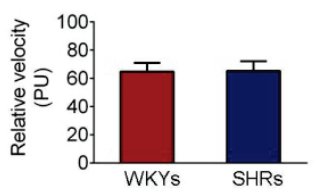

Fig. 1. Pancreatic microcirculation profiles of WKYs and SHRs. (A) pancreatic microcirculatory blood distribution pattern of WKYs and SHRs. (B) pancreatic microvascular vasomotion of two groups of rats. (C) velocity of microvascular vasomotion. (D) 5 secs microvascular blood flow perfusion extracted from microcirculatory blood perfusion between dashed lines. (E) the average blood perfusion (PU/min) of microvascular vasomotion. (F) the amplitude ( $\triangle \mathrm{PU}$ ) was calculated as the difference between minimum PU and maximum PU in microvascular oscillation. (G) the number of peaks in microvascular oscillation per min was defined as frequency (cycles/min). (H) microcirculatory velocity between two groups. Red dots and curves represent blood perfusion of WKYs. Blue dots and curves represent blood flow perfusion of SHRs. Green dashed line, the cut-off line of distribution pattern between SHRs and WKYs. PU, perfusion units. ${ }^{*} P<0.05$ compared with WKYs, ${ }^{*} P<0.01$ compared with WKYs.

\section{Results}

The general information about SHRs and their normotensive control is summarized in Table 1. To assess whether pancreatic microcirculation profiles were abnormal in SHRs, we generated microcirculatory blood distribution patterns. With the normotensive condition, WKYs presented a higher scale of blood perfusion pattern, in contrast, SHRs exhibited a lower scale of blood perfusion pattern (Fig. 1A), suggesting 

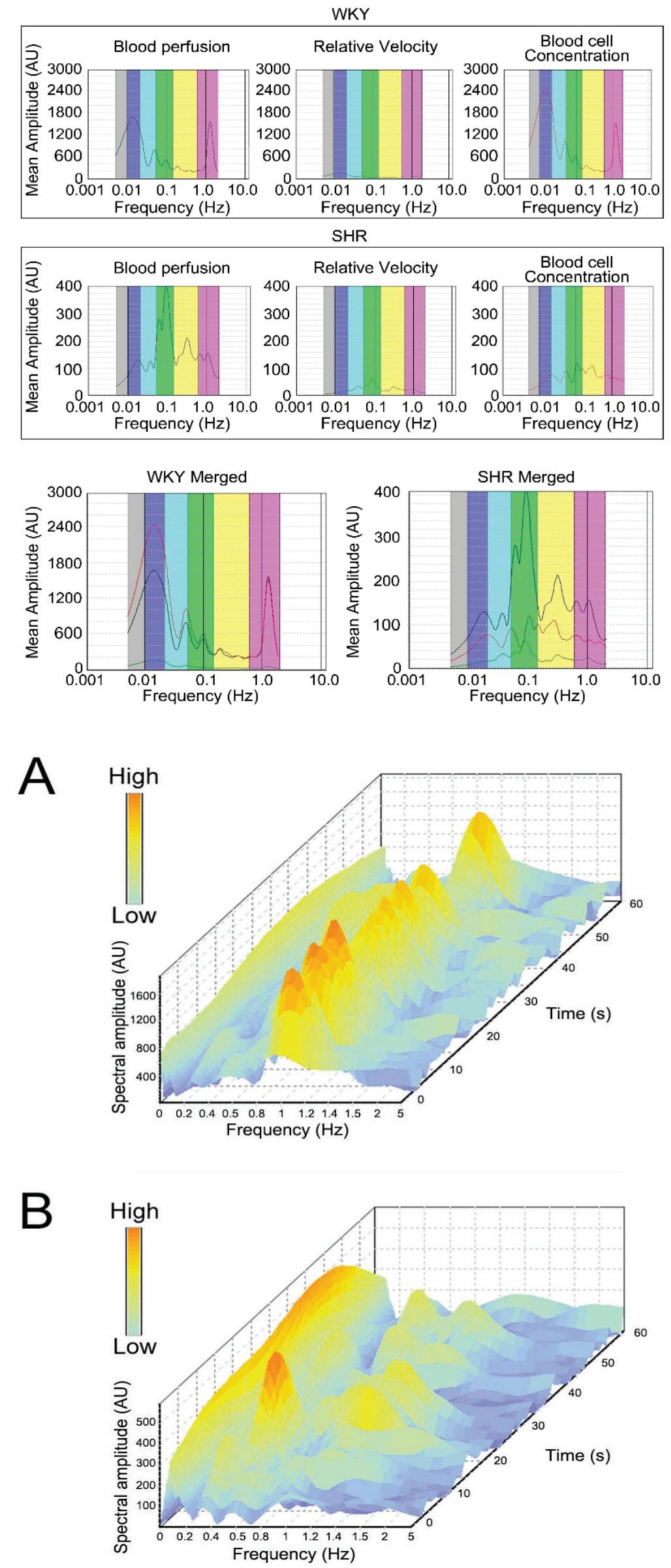

Fig. 2. Wavelet transform spectral analysis of pancreatic micro-hemodynamic signals. The mean amplitude $(\mathrm{AU})$ - frequency $(\mathrm{Hz})$ spectrum of microcirculatory blood perfusion, relative velocity and blood cell concentration was revealed by wavelet transform spectral analysis. The merged pancreatic microcirculatory scalogram of WKYs and SHRs was exhibited in the lower panel, the vertical lines represent the cut-off boundaries of different frequency intervals.

Fig. 3. Three-dimensional time-frequency spectral scalogram of pancreatic microcirculation profiles of WKYs and SHRs. (A-B) threedimensional time-frequency spectral scalogram of WKYs and SHRs were constructed based on the micro-hemodynamics data. The microvascular blood perfusion signaling was transformed by wavelet coefficients to illustrate coordinated time-frequency resolution in SHRs (A) and WKYs (B). Micro-hemodynamic variants including time $(\mathrm{s})$, frequency $(\mathrm{Hz})$ and spectral amplitude (AU) were located in the coordinate respectively. Color bar represents amplitude values. 
a divergence of microcirculatory blood distribution pattern between WKYs and SHRs. The bio-rhythmic contraction and dilatation of pancreatic microcirculation (vasomotion) were disarranged in SHRs, while WKYs displayed stable bio-rhythmic contraction and relaxation (Fig. 1B-1D). Quantitative analysis revealed that the micro-hemodynamic parameters including average blood perfusion $(P<0.05$, Fig. 1E), amplitude $(P<0.05$, Fig. $1 \mathrm{~F})$ and frequency $(P<0.01$, Fig. $1 G)$ of microvascular vasomotion were significantly decreased in SHRs. However, no significant difference of velocity was found across microvascular oscillation between two groups (Fig. 1H). Taken together, our data indicate deteriorated pancreatic microcirculation profiles in SHRs.

The comparisons of wavelet transformed microhemodynamic signals were illustrated as amplitudefrequency scalogram in Figure 2. The scalogram revealed the contribution of endothelial oscillators make to biorhythmic micro-hemodynamic in hypertensive and normotensive animals. Compared with WKYs, the characteristic peak amplitudes of blood perfusion, relative velocity and blood cell concentration were significantly decreased in endothelial oscillators in SHRs. Furthermore, after embedding the time course dimension, we generated 3-D time-frequency spectral scalogram. It was noted that WKYs and SHRs exhibited different timefrequency spectral pattern. SHRs exhibited reduced endothelial amplitudes in pancreatic microcirculatory oscillation (Fig. 3). We then compared the amplitudes of the characteristic non-endothelial and endothelial oscillators between SHRs and WKYs.

Furthermore, our data revealed that the amplitudes of endothelial oscillators were significantly decreased in SHRs (Fig. 4A). Considering microhemodynamic abnormalities are the global pathological microcirculatory phenotype rather than specific single characteristic oscillator, we then integrated amplitudes of characteristic oscillators into radar map (Fig. 4B). The radar map illustrated that different amplitude regimes separated by endothelial NO-dependent and NO-independent oscillators between SHRs and WKYs (Fig. 4C). Since the endothelial oscillators of pancreatic
A

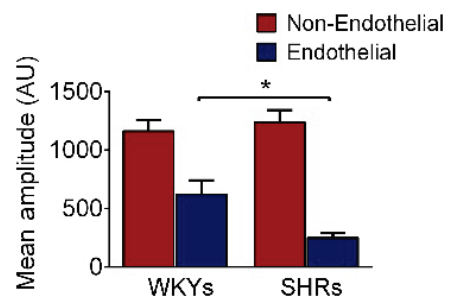

$\mathrm{B}$

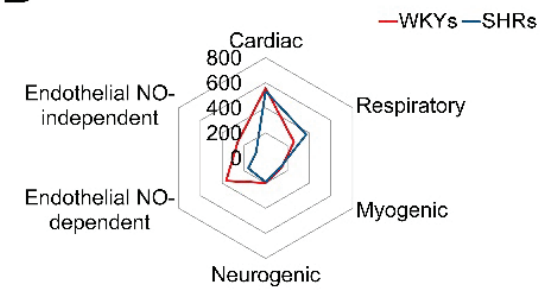

D

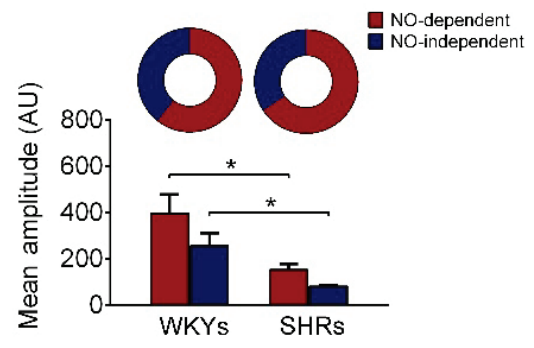

$\mathrm{E}$
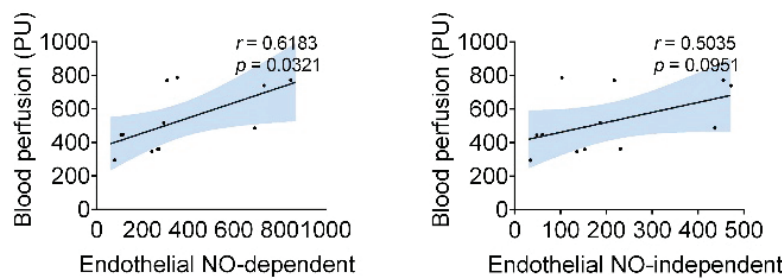

Fig. 4. Comparisons of characteristic spectral amplitudes of pancreatic microcirculation profiles. (A) the mean amplitudes (AU) of nonendothelial components and endothelial components of WKYs and SHRs. (B) radar plot illustrated varying amplitudes distribution of characteristic oscillators. The red line represents amplitudes of WKYs, the blue line represents amplitudes of SHRs. (C) the mean amplitudes (AU) of characteristic oscillators. (D) comparisons of NO-dependent and NO-independent endothelial components between WKYs and SHRs. The ratios of NO-dependent and NO-independent endothelial oscillators were shown in the upper pie chart. (E) correlations between endothelial oscillators and microcirculatory blood perfusion. ${ }^{*} P<0.05$ compared with WKYs. 
A

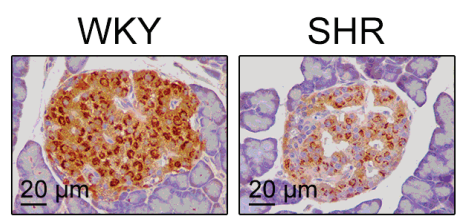

C



B

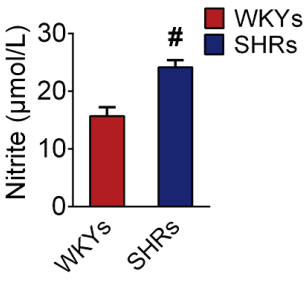

D

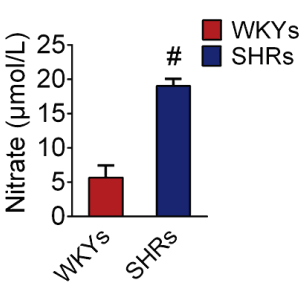

$\mathrm{E}$
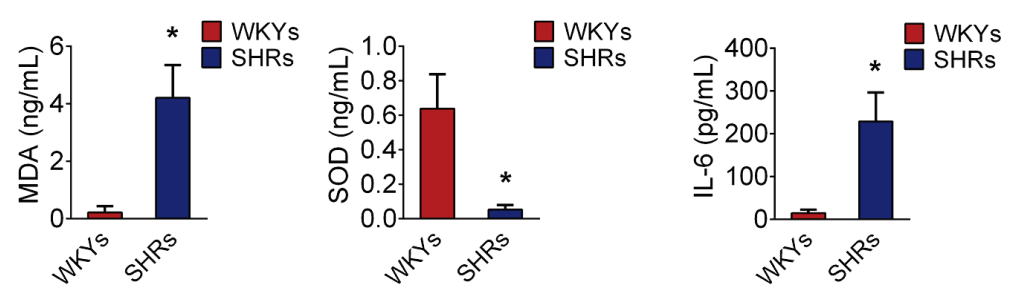

Fig. 5. Pancreatic microvascular endothelial cells dysfunction in SHRs. (A) immunohistochemical staining of endothelial nitric oxide synthase in WKYs and SHRs $(\times 400)$. (B) the levels of plasma nitrite/nitrate of WKYs and SHRs. (C) plasma endothelin-1 of WKYs and SHRs. (D) plasma MDA and SOD levels. (E) plasma IL-6 of WKYs and SHRs. ${ }^{*} P<0.05$ compared with WKYs, ${ }^{*} P<0.01$ compared with WKYs.

microcirculation profiles were deteriorated, the mean amplitudes of NO-dependent and NO-independent endothelial oscillators in SHRs and WKYs were further analyzed. As expect, we found significant decreased mean amplitudes of both NO-dependent and NO-independent endothelial oscillators in SHRs compared with WKYs (Fig. 4D). Additionally, the difference amplitude value $(\triangle \mathrm{AU})$ of NO-dependent endothelial oscillator $(965.30 \mathrm{AU})$ was significantly larger than NO-independent endothelial component (592.80 AU) in SHRs. Accordingly, a positive correlation between the amplitude of NO-dependent endothelial oscillator and microcirculatory blood perfusion $(r=0.6183, P<0.05)$ was observed, whereas no correlation was found between NO-independent endothelial oscillator $(r=0.5035, P>0.05)$ and microvascular blood perfusion (Fig. 4E). This is consistent with findings from wavelet transform analysis, where endothelial oscillators may be involved in hypertensive responses.

Microvascular endothelial dysfunction is characterized by imbalanced microcirculatory tone which is associated with eNOS. Based on the pancreatic microhemodynamic disturbances observed in SHRs, we therefore queried whether the eNOS expression, plasma nitrite/nitrate and ET-1 levels were abnormal. Immunostaining analysis revealed a decreased expression of eNOS in SHRs (Fig. 5A). To further determine the endothelial function, we evaluated the plasma nitrite/nitrate and ET-1 levels. As shown in Figure 5B, in addition to significantly increased plasma nitrite/nitrate level, as a vasoconstrictor, ET-1 was significantly increased in SHRs compared with their normotensive control (Fig. 5C). Moreover, the antioxidant status of SHRs was deteriorated, which included significantly increased plasma MDA level and decreased SOD activity (Fig. 5D). Meanwhile, SHRs exhibited an elevation of inflammatory cytokine IL-6 level (Fig. 5E).

\section{Discussion}

Microcirculation regulates blood distribution and perfusion via microvascular vasomotion so as to maintain the physiological functions of organs (Segal 2005). Emerging evidence has indicated that pancreas is a potential source (Chan and Schmid-Schonbein 2019) for leaked serine proteases (Derosa et al. 2006), which contributes to uncontrolled protease activities in hypertensive individuals. A number of experimental data, including ours, have been proposed to explain this hypothesis observed in SHRs through the pancreatic microcirculation dysfunction. The intravital microscopic findings provide the evidence for the leakage of macromolecular FITC-dextran from the pancreatic microvasculature into the interstitial tissue (Zhou and Chen 2002). Furthermore, leakage of the cast material through the pancreatic capillary membrane suggest that presence of increased permeability during the pathological process (Eibl et al. 2000). In this study, we showed that compared with WKYs, the pancreatic microcirculation profiles of SHRs were abnormal, which 
exhibited disarranged blood distribution pattern, impaired microvascular vasomotion and reduced amplitudes of endothelial oscillators.

Several studies have confirmed that malfunctional microcirculation is involved in the pathogenesis of hypertension in vitro and in vivo. It was reported that the capacities of arterioles regulating flow resistance were abnormal in hypertensive individuals (Martinez-Lemus 2012). Additionally, terminal resistance microvessels remodeling (Risler et al. 2005), capillary rarefaction and stiffness (Serne et al. 2001) and microvasculature alterations may contribute to the development of hypertension. Besides these structural evidence, we provided microcirculatory data concerning the occurrence of impaired pancreatic microcirculation profiles in SHRs.

Microvascular vasomotion is one of the essential properties of microcirculation profiles for the maintenance of homeostasis. Microvascular vasoconstriction and vasodilation regulate blood perfusion and distribution pattern of organs (Liu et al. 2017a). As expected, we demonstrated that SHRs exhibited disarranged microvascular vasomotion and failed to maintain bio-rhythmic blood perfusion which hampered the exchange of metabolic nutrients. On the other hand, it is also noteworthy that capillary rarefaction (decreased microvessels density) has been demonstrated occurring in SHRs (Wang et al. 2014), which may contribute to the decreased blood perfusion and disrupted delivery of nutrients and oxygen (Plotnikov et al. 2018, Suzuki et al. 2003). Consequently, micro-hemodynamic disorders may be one of the explanations for the decreased microvascular blood perfusion.

Microcirculatory oscillation is affected by a series of vasomotor stemming from the characteristic oscillators of these micro-vessels, especially microvascular endothelial cells. Thus, the characteristic oscillators are considered as dominant determinants of pancreatic microcirculation. As indicated in this study, the decreased NO-dependent endothelial oscillator, rather than other components, may be associated with hypertensive pathological condition. Supporting the importance of microcirculation profiles, our data revealed the specific oscillators of pancreatic microcirculatory abnormalities in hypertensive rats, which is consistent with confirmed impaired barrier function, inhibited migration and tube formation capacities of pancreatic microvascular endothelial cells (Liu et al. 2017b).

Inflammatory cascade and oxidative stress are recognized as two crucial pathological processes in the development of hypertension (Khullar et al. 2004, Redon et al. 2003). Due to the deteriorated endothelial oscillators, micro-hemodynamics tends to be proconstrictive, pro-thrombotic and anti-fibrinolytic (Rosenblum 2018), leading to reactive oxygen species generation and inflammatory cytokine synthesis. Meanwhile, increased oxidative stress and activated aggravating cytokines could occur in hypertensive individuals (Domingueti et al. 2016, Trejo-Moreno et al. 2018). It has been reported that eNOS contributed to vasodilation and related to the pathological determinants such as oxidative stress and inflammatory cytokines (Forstermann and Munzel 2006). Reduction (Kloza et al. 2019) and inactivation (Peleli et al. 2016) of eNOS in hypertensive rat might mediate vasodilatory dysfunction. NO, synthesized by eNOS, is an endothelial-derived vasodilator, which maintains microvascular endothelial physiological function via nitrate-nitrite-NO pathway (Victor et al. 2009). Consistently, the present study showed reduced expression of eNOS and decreased amplitudes of NO-independent and NO-dependent endothelial oscillators in SHRs, together with increased nitrate/nitrite and ET-1 levels, which may result in the decrease of flow-mediated vasodilation (Kong et al. 2015, Nickenig et al. 2000, Gradin et al. 2018). And increased levels of MDA, IL- 6 and decreased levels of SOD lead to destabilized eNOS and incapable availability of NO, which might be responsible for the observed deteriorated pancreatic microcirculation profiles. Additionally, it has been reported that the inhibition of SOD promotes vasoconstriction (Ahmeda et al. 2018), confirming that oxidative stress is strongly associated with hypertension from another perspective. Our data further strengthens the importance of endothelial oscillators and pancreatic microcirculation profiles in hypertension as well.

In conclusion, our study provides evidence that SHRs exhibit abnormal pancreatic microcirculation profiles including disarranged pancreatic blood distribution pattern, impaired pancreatic microvascular vasomotion and reduced amplitudes of endothelial oscillators.

\section{Conflict of Interest}

There is no conflict of interest.

\section{Acknowledgements}

This work was supported by grants from the CAMS 
Initiative for Innovative Medicine (CAMS-I2M) (No.

Foundation (No. 81900747).

2016-I2M-3-006) and the National Natural Science

\section{References}

AHMEDA AF, RAE MG, ANWEIGI LM, AL OTAIBI MF, AL-MASRI AA, JOHNS EJ: The effect of superoxide dismutase enzyme inhibition on renal microcirculation of spontaneously hypertensive-stroke prone and Wistar rats. Physiol Res 67: 535-541, 2018. https://doi.org/10.33549/physiolres.933655

ALEKSANDRIN VV, IVANOV AV, VIRUS ED, BULGAKOVA PO, KUBATIEV AA: Application of wavelet analysis to detect dysfunction in cerebral blood flow autoregulation during experimental hyperhomocysteinaemia. Lasers Med Sci 33: 1327-1333, 2018. https://doi.org/10.1007/s10103-018-2485-x

CHAN AHW, SCHMID-SCHONBEIN GW: Pancreatic source of protease activity in the spontaneously hypertensive rat and its reduction during temporary food restriction. Microcirculation 26: e12548, 2019. https://doi.org/10.1111/micc. 12548

DALYS GBD, COLLABORATORS H: Global, regional, and national disability-adjusted life-years (DALYs) for 315 diseases and injuries and healthy life expectancy (HALE), 1990-2015: a systematic analysis for the Global Burden of Disease Study 2015. Lancet 388: 1603-1658, 2016.

DE BOER MP, MEIJER RI, WIJNSTOK NJ, JONK AM, HOUBEN AJ, STEHOUWER CD, SMULDERS YM, ERINGA EC, SERNE EH: Microvascular dysfunction: a potential mechanism in the pathogenesis of obesityassociated insulin resistance and hypertension. Microcirculation 19: 5-18, 2012. https://doi.org/10.1111/j.15498719.2011.00130.x

DEBBABI H, UZAN L, MOURAD JJ, SAFAR M, LEVY BI, TIBIRICA E: Increased skin capillary density in treated essential hypertensive patients. Am J Hypertens 19: 477-483, 2006. https://doi.org/10.1016/j.amjhyper.2005.10.021

DELANO FA, SCHMID-SCHONBEIN GW: Proteinase activity and receptor cleavage: mechanism for insulin resistance in the spontaneously hypertensive rat. Hypertension 52: 415-423, 2008. https://doi.org/10.1161/HYPERTENSIONAHA.107.104356

DELANO FA, ZHANG H, TRAN EE, ZHANG C, SCHMID-SCHONBEIN GW: A new hypothesis for insulin resistance in hypertension due to receptor cleavage. Expert Rev Endocrinol Metab 5: 149-158, 2010. https://doi.org/10.1586/eem.09.64

DEROSA G, D'ANGElO A, CICCARELli L, PICCINNI MN, PRICOLO F, SALVADEO S, MONTAGNA L, GRAVINA A, FERRARI I, GALLI S, PANIGA S, TINELLI C, CICERO AF: Matrix metalloproteinase-2, -9, and tissue inhibitor of metalloproteinase-1 in patients with hypertension. Endothelium 13: 227-231, 2006. https://doi.org/10.1080/10623320600780942

DOMINGUETI CP, DUSSE LM, CARVALHO M, DE SOUSA LP, GOMES KB, FERNANDES AP: Diabetes mellitus: The linkage between oxidative stress, inflammation, hypercoagulability and vascular complications. J Diabetes Complications 30: 738-745, 2016. https://doi.org/10.1016/j.jdiacomp.2015.12.018

EIBL G, HOTZ HG, FAULHABER J, KIRCHENGAST M, BUHR HJ, FOITZIK T: Effect of endothelin and endothelin receptor blockade on capillary permeability in experimental pancreatitis. Gut 46: 390-394, 2000. https://doi.org/10.1136/gut.46.3.390

FORSTERMANN U, MUNZEL T: Endothelial nitric oxide synthase in vascular disease: from marvel to menace. Circulation 113: 1708-1714, 2006. https://doi.org/10.1161/CIRCULATIONAHA.105.602532

GKALIAGKOUSI E, GAVRIILAKI E, TRIANTAFYLLOU A, DOUMA S: Clinical significance of endothelial dysfunction in essential hypertension. Curr Hypertens Rep 17: 85, 2015. https://doi.org/10.1007/s11906-0150596-3

GRADIN K, PERSSON B: Endothelin A receptor blockade improves endothelium-dependent relaxation in obese woman. Physiol Res 67: S167-S174, 2018. https://doi.org/10.33549/physiolres.933813 
GUTTERMAN DD, CHABOWSKI DS, KADLEC AO, DURAND MJ, FREED JK, AIT-AISSA K, BEYER AM: The human microcirculation: regulation of flow and beyond. Circ Res 118: 157-172, 2016. https://doi.org/10.1161/CIRCRESAHA.115.305364

KHULLAR M, RELAN V, SEHRAWAT BS: Antioxidant activities and oxidative stress byproducts in human

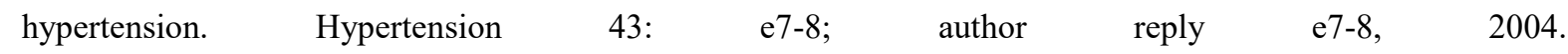
https://doi.org/10.1161/01.HYP.0000111210.34843.b6

KLOZA M, BARANOWSKA-KUCZKO M, TOCZEK M, KUSACZUK M, SADOWSKA O, KASACKA I, KOZLOWSKA H: Modulation of cardiovascular function in primary hypertension in rat by SKA-31, an activator of KCa2.x and KCa3.1 channels. Int J Mol Sci 20: 4118, 2019. https://doi.org/10.3390/ijms20174118

KOBAYASHI N, DELANO FA, SCHMID-SCHONBEIN GW: Oxidative stress promotes endothelial cell apoptosis and loss of microvessels in the spontaneously hypertensive rats. Arterioscler Thromb Vasc Biol 25: 2114-2121, 2005. https://doi.org/10.1161/01.ATV.0000178993.13222.f2

KONG X, LI W, GUO LQ, ZHANG JX, CHEN XP, LIU WY, YANG JR: Sesamin enhances nitric oxide bioactivity in aortas of spontaneously hypertensive rats. Ther Adv Cardiovasc Dis 9: 314-324, 2015. https://doi.org/10.1177/1753944715586178

LANCASTER G, STEFANOVSKA A, PESCE M, MARCO VEZZONI G, LOGGINI B, PINGITORE R, GHIARA F, BARACHINI P, CERVADORO G, ROMANELLI M, ROSSI M: Dynamic markers based on blood perfusion fluctuations for selecting skin melanocytic lesions for biopsy. Sci Rep 5: 12825, 2015. https://doi.org/10.1038/srep12825

LAPI D, MASTANTUONO T, DI MARO M, VARANINI M, COLANTUONI A: Low-frequency components in rat pial arteriolar rhythmic diameter changes. J Vasc Res 54: 344-358, 2017. https://doi.org/10.1159/000478984

LEVY BI, AMBROSIO G, PRIES AR, STRUIJKER-BOUDIER HA: Microcirculation in hypertension: a new target for treatment? Circulation 104: 735-740, 2001. https://doi.org/10.1161/hc3101.091158

LIU M, ZHANG X, LI A, ZHANG X, WANG B, LI B, LIU S, LI H, XIU R: Insulin treatment restores islet microvascular vasomotion function in diabetic mice. $J$ Diabetes 9: 958-971, $2017 \mathrm{a}$. https://doi.org/10.1111/1753-0407.12516

LIU M, ZHANG X, WANG B, WU Q, LI B, LI A, ZHANG H, XIU R: Functional status of microvascular vasomotion is impaired in spontaneously hypertensive rat. Sci Rep 7: 17080, 2017b. https://doi.org/10.1038/s41598-01717013-W

LIU M, ZHANG X, LI B, WANG B, WU Q, SHANG F, LI A, LI H, XIU R: Laser Doppler: a tool for measuring pancreatic islet microvascular vasomotion in vivo. J Vis Exp 3: e56028, 2018. https://doi.org/10.3791/56028

MARTINEZ-LEMUS LA: The dynamic structure of arterioles. Basic Clin Pharmacol Toxicol 110: 5-11, 2012. https://doi.org/10.1111/j.1742-7843.2011.00813.x

MASTANTUONO T, STARITA N, BATTILORO L, DI MARO M, CHIURAZZI M, NASTI G, MUSCARIELLO E, CESARELLI M, IUPPARIELLO L, D'ADDIO G, GORBACH A, COLANTUONI A, LAPI D: Laser speckle imaging of rat pial microvasculature during hypoperfusion-reperfusion damage. Front Cell Neurosci 11: 298, 2017. https://doi.org/10.3389/fncel.2017.00298

NICKENIG G, STABLEIN A, WASSMANN S, WYEN C, MULLER C, BOHM M: Acute effects of ACE inhibition on coronary endothelial dysfunction. J Renin Angiotensin Aldosterone Syst 1: 361-364, 2000. https://doi.org/10.3317/jraas.2000.067

PELELI M, ZOLLBRECHT C, MONTENEGRO MF, HEZEL M, ZHONG J, PERSSON EG, HOLMDAHL R, WEITZBERG E, LUNDBERG JO, CARLSTROM M: Enhanced XOR activity in eNOS-deficient mice: Effects on the nitrate-nitrite-NO pathway and ROS homeostasis. Free Radic Biol Med 99: 472-484, 2016. https://doi.org/10.1016/j.freeradbiomed.2016.09.004

PLOTNIKOV MB, ALIEV OI, SIDEKHMENOVA AV, SHAMANAEV AY, ANISHCHENKO AM, FOMINA TI, PLOTNIKOVA TM, ARKHIPOV AM: Effect of p-tyrosol on hemorheological parameters and cerebral capillary network in young spontaneously hypertensive rats. Microvasc Res 119: 91-97, 2018. https://doi.org/10.1016/j.mvr.2018.04.005 
POPA SO, FERRARI M, ANDREOZZI GM, MARTINI R, BAGNO A: Wavelet analysis of skin perfusion to assess the effects of FREMS therapy before and after occlusive reactive hyperemia. Med Eng Phys 37: 1111-1115, 2015. https://doi.org/10.1016/j.medengphy.2015.08.016

REDON J, OLIVA MR, TORMOS C, GINER V, CHAVES J, IRADI A, SAEZ GT: Antioxidant activities and oxidative stress byproducts in human hypertension. Hypertension 41: 1096-1101, 2003. https://doi.org/10.1161/01.HYP.0000068370.21009.38

RISLER NR, CRUZADO MC, MIATELLO RM: Vascular remodeling in experimental hypertension. ScientificWorldJournal 5: 959-971, 2005. https://doi.org/10.1100/tsw.2005.122

ROSENBLUM WI: Endothelium-dependent responses in the microcirculation observed in vivo. Acta Physiol (Oxf) 224: e13111, 2018. https://doi.org/10.1111/apha.13111

SEGAL SS: Regulation of blood flow in the microcirculation. Microcirculation 12: 33-45, 2005. https://doi.org/10.1080/10739680590895028

SERNE EH, GANS RO, TER MAATEN JC, TANGELDER GJ, DONKER AJ, STEHOUWER CD: Impaired skin capillary recruitment in essential hypertension is caused by both functional and structural capillary rarefaction. Hypertension 38: 238-242, 2001. https://doi.org/10.1161/01.HYP.38.2.238

SMIRNI S, MACDONALD MP, ROBERTSON CP, MCNAMARA PM, O'GORMAN S, LEAHY MJ, KHAN F: Application of cmOCT and continuous wavelet transform analysis to the assessment of skin microcirculation dynamics. J Biomed Opt 23: 1-13, 2018. https://doi.org/10.1117/1.JBO.23.7.076006

STEFANOVSKA A, BRACIC M, KVERNMO HD: Wavelet analysis of oscillations in the peripheral blood circulation measured by laser Doppler technique. IEEE Trans Biomed Eng 46: 1230-1239, 1999. https://doi.org/10.1109/10.790500

STRUIJKER-BOUDIER HA, ROSEI AE, BRUNEVAL P, CAMICI PG, CHRIST F, HENRION D, LEVY BI, PRIES A, VANOVERSCHELDE JL: Evaluation of the microcirculation in hypertension and cardiovascular disease. Eur Heart J 28: 2834-2840, 2007. https://doi.org/10.1093/eurheartj/ehm448

SUZUKI K, MASAWA N, SAKATA N, TAKATAMA M: Pathologic evidence of microvascular rarefaction in the brain of renal hypertensive rats. J Stroke Cerebrovasc Dis 12: 8-16, 2003. https://doi.org/10.1053/jscd.2003.1

TOUSOULIS D, ANTONIADES C, KOUMALLOS N, MARINOU K, STEFANADI E, LATSIOS G, STEFANADIS $\mathrm{C}$ : Novel therapies targeting vascular endothelium. Endothelium 13: 411-421, 2006. https://doi.org/10.1080/10623320601061714

TRAN ED, SCHMID-SCHONBEIN GW: An in-vivo analysis of capillary stasis and endothelial apoptosis in a model of hypertension. Microcirculation 14: 793-804, 2007. https://doi.org/10.1080/10739680701419992

TREJO-MORENO C, MENDEZ-MARTINEZ M, ZAMILPA A, JIMENEZ-FERRER E, PEREZ-GARCIA MD, MEDINA-CAMPOS ON, PEDRAZA-CHAVERRI J, SANTANA MA, ESQUIVEL-GUADARRAMA FR, CASTILLO A, CERVANTES-TORRES J, FRAGOSO G, ROSAS-SALGADO G: Cucumis sativus aqueous fraction inhibits angiotensin II-induced inflammation and oxidative stress in vitro. Nutrients 10: E276, 2018. https://doi.org/10.3390/nu10030276

VICTOR VM, NUNEZ C, D'OCON P, TAYLOR CT, ESPLUGUES JV, MONCADA S: Regulation of oxygen distribution in tissues by endothelial nitric oxide. Circ Res 104: 1178-1183, 2009.https://doi.org/10.1161/CIRCRESAHA.109.197228

WANG B, LI BW, LI HW, LI AL, YUAN XC, WANG Q, XIU RJ: Enhanced matrix metalloproteinases-2 activates aortic endothelial hypermeability, apoptosis and vascular rarefaction in spontaneously hypertensive rat. Clin Hemorheol Microcirc 57: 325-338, 2014. https://doi.org/10.3233/CH-131713

ZHOU ZG, CHEN YD: Influencing factors of pancreatic microcirculatory impairment in acute panceatitis. World J Gastroenterol 8: 406-412, 2002. https://doi.org/10.3748/wjg.v8.i3.406 